\title{
New Dimensions of Research on Actinomycetes: Quest for Next Generation Antibiotics
}

\author{
Polpass Arul Jose ${ }^{1 *}$ and Bhavanath Jha ${ }^{1,2 *}$ \\ ${ }^{1}$ Marine Biotechnology and Ecology Division, CSIR - Central Salt and Marine Chemicals Research Institute, Bhavnagar, \\ India, ${ }^{2}$ Academy of Scientific and Innovative Research (AcSIR), Council of Scientific and Industrial Research, New Delhi, India
}

OPEN ACCESS

Edited by:

Jem Stach,

Newcastle University, UK

Reviewed by:

Nicholas Allenby,

Demuris Ltd, UK

Osmar Nascimento Silva, Universidade Católica Dom Bosco,

Brazil

*Correspondence:

Bhavanath Jha

bjha@csmcri.org

Polpass Arul Jose

arulmku@gmail.com

Specialty section: This article was submitted to Antimicrobials, Resistance and Chemotherapy,

a section of the journal

Frontiers in Microbiology

Received: 21 May 2016 Accepted: 05 August 2016 Published: 19 August 2016

Citation:

Jose PA and Jha B (2016) New

Dimensions of Research on Actinomycetes: Quest for Next

Generation Antibiotics.

Front. Microbiol. 7:1295.

doi: 10.3389/fmicb.2016.01295
Starting with the discovery of streptomycin, the promise of natural products research on actinomycetes has been captivating researchers and offered an array of lifesaving antibiotics. However, most of the actinomycetes have received a little attention of researchers beyond isolation and activity screening. Noticeable gaps in genomic information and associated biosynthetic potential of actinomycetes are mainly the reasons for this situation, which has led to a decline in the discovery rate of novel antibiotics. Recent insights gained from genome mining have revealed a massive existence of previously unrecognized biosynthetic potential in actinomycetes. Successive developments in next-generation sequencing, genome editing, analytical separation and high-resolution spectroscopic methods have reinvigorated interest on such actinomycetes and opened new avenues for the discovery of natural and naturalinspired antibiotics. This article describes the new dimensions that have driven the ongoing resurgence of research on actinomycetes with historical background since the commencement in 1940, for the attention of worldwide researchers. Coupled with increasing advancement in molecular and analytical tools and techniques, the discovery of next-generation antibiotics could be possible by revisiting the untapped potential of actinomycetes from different natural sources.

Keywords: actinomycetes, natural products, antibiotics, drug discovery, genomics, metabolomics

\section{INTRODUCTION}

Actinomycetes are ubiquitous Gram-positive bacteria that constitute one of the largest bacterial phyla with characteristic filamentous morphology and high G+C DNA. The actinomycetes have been recognized as premier source and inspiration for a substantial fraction of antibiotics that play an important role in human health. The most striking fact is that these filamentous bacteria have 


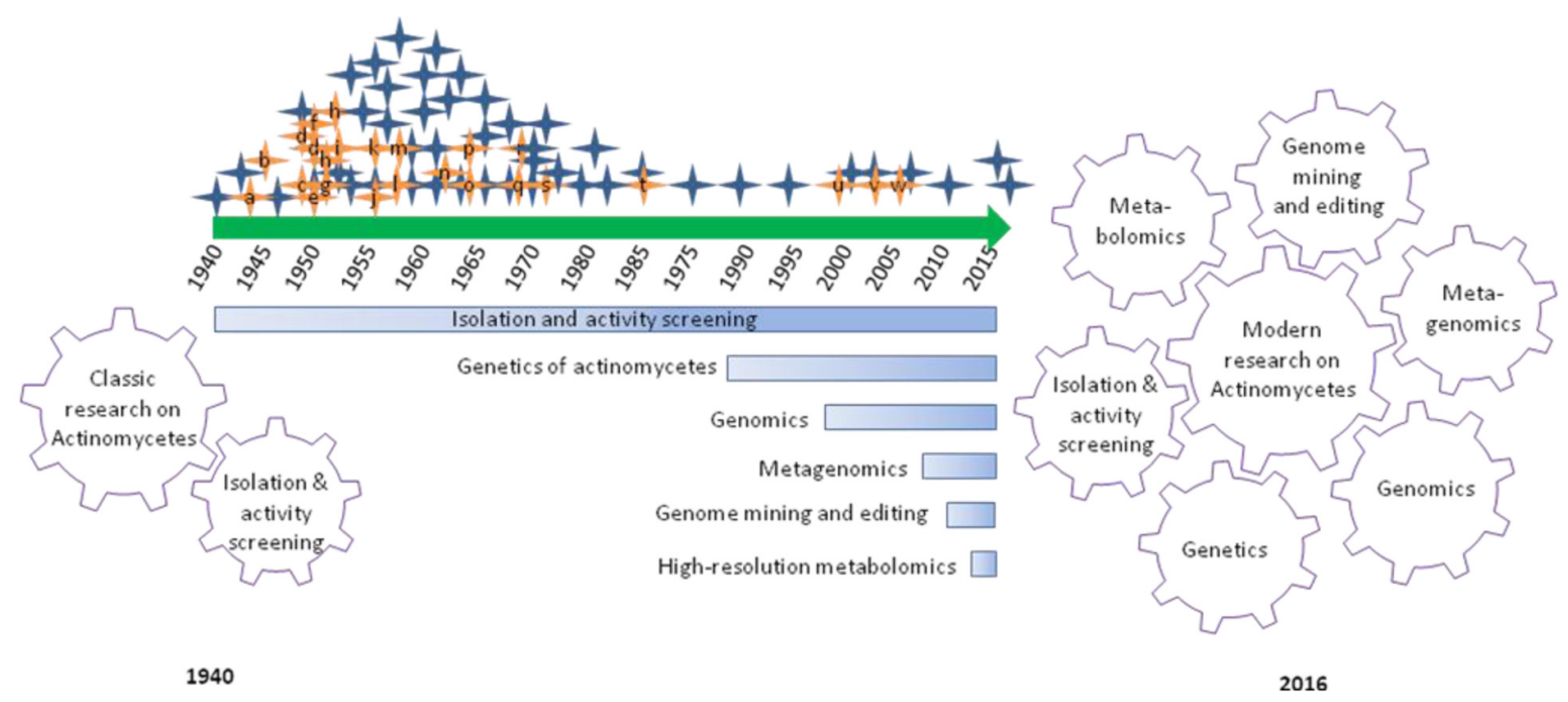

FIGURE 1 | Graphical summary of research and developments focused on antibiotic discovery from actinomycetes over 76 years. Hunting of antibiotics from actinomycetes has emanated with the discovery of actinomycin in 1940 (a) and lined up with several commercially important antibiotics and their derivatives: streptomycin (a), cephalosporins (b), Chloramphenicol (c), neomycin (d), tetracycline (e), nystatin (f), virginiamycin (g), erythromycin (h), lincomycin (i), vancomycin (j), noviobiocin (k), rifamycin (l), kanamycin (m), nalidixic acid (n), fusidic acid (o), gentamicin (p), trimethoprim (q), fostomycin (r), ribostamycin (s), mupiriocin (t), linezolid (u), daptomycin (v), and platensimycin (w). Classic actinomycetes research was driven by isolation and activity screening approach. Whereas, modern actinomycetes research is driven by array of breakthroughs in genetics, genomics, metagenomics, genome mining and editing and high-resolution metabolomics, in association with classical approach.

evolved with the wealth of biosynthetic gene clusters and thereby show an unprecedented potential in production biologically active natural product scaffolds. However, last two decades has seen a move by pharmaceutical giants away from microbial natural product discovery efforts, and such efforts continue to flourish in research institutes with promising results. The continued research efforts of academic research institutes, with post-genomic technological innovations, rejuvenate natural product research and compose a clarion call to worldwide researchers for tuning into microbial natural products research.

\section{THE CLASSIC ACTINOMYCETES RESEARCH}

If we look back to about 76 years of actinomycetes research that focused on hunting bioactive metabolites of public welfare, over 5000 compounds have been reported and contributed to the development of $90 \%$ of commercial antibiotics being used for either clinical or research needs. In this long course, actinomycetes research evolved several aspects from isolation and activity screening to modern post-genomic secondary metabolites research (Figure 1). The first report of streptomycin by Selman Waksman and associates in the 1940s and subsequent development as drug encouraged pharmaceutical companies and researchers to put their large scale efforts on microbial natural products research (Demain and Sanchez, 2009). The efforts were largely depending on the recovery of microorganisms from diverse environmental samples, and screening for the desired bioactivity. The approach brought the golden era (1950-1970) of antibiotic discovery evidenced by the commercialization of several life-saving antibiotics including streptomycin, vancomycin, rifamycin, and so on (Mahajan and Balachandran, 2012). In subsequent decades, the rediscovery of known compounds and technical challenges associated with purification and structure elucidation of new compounds largely declined the classic efforts (Bérdy, 2012). Despite the evidence of a decline in microbial natural products research, continued innovations in sampling and acquisition of potential actinomycetes from previously unexplored sources are being continued by several academic research groups and mitigate risks of the rediscovery of known compounds and augmented availability of diverse actinomycetes that are fundamental matters to the long term actinomycetes research.

\section{IN PROGRESS}

Progress is crucial in several aspects of actinomycetes research that includes (1) isolation and dereplication of actinomycete isolates, (2) prediction and identification novel compounds, (3) enhancing production titers of potential compounds, (4) uncovering genome information and associated biosynthetic potential, (5) collection and processing of genomic data, (6) mining, editing and heterologous expression of cryptic gene clusters, and (7) comprehensive metabolic profiling, under a broad spectrum of main areas such as genetics, genomics and metabolomics.

Establishing actinomycete resources is one of the basic requirements for culture-dependent natural products research. 
To address this, researchers are learning how to cultivate the unexplored actinomycete biodiversity in diverse environments and such efforts have led to cultivation of numerous novel actinomycetes from marine sediments (Becerril-Espinosa et al., 2013), hydrothermal vents (Thornburg et al., 2010), solar salterns (Jose and Jebakumar, 2013), desert soils (Mohammadipanah and Wink, 2016), red soils (Guo et al., 2015), sponges (Sun et al., 2015), insects (Matsui et al., 2012; Kurtböke et al., 2015), and plants (Masand et al., 2015). On the other hand, dereplication of isolated strains has attained a new pitch with gene specific as well as metabolic fingerprinting approaches (Hou et al., 2012; Forner et al., 2013). Collectively, the united success in isolation and dereplication facilitates the prioritization of the isolates which could be cellular factories with the innate biosynthetic capability to produce novel compounds. One such approach has been practiced to isolate 64 distinctive actinomycetes from 12 different marine sponge species, and to prioritize two unique strains that showed anti-trypanosomal activity as well as uniqueness in metabolomic profile and richness of unidentified natural products (Cheng et al., 2015).

Prediction and identification of novel compounds from actinomycetes including those with low production titers have become relatively straight forward through the advent of highresolution liquid chromatography-mass spectrometry (HR-LCMS) and allied database search (Tawfike et al., 2013; Doroghazi et al., 2014; Wu et al., 2016). Recently, Wu et al. (2016) were able to demonstrate the employability of NMR-based metabolic profiling method to streamline microbial biotransformation and to determine the best harvesting time of actinomycetes for antibiotic production. Technical breakthroughs also in gene level understanding and recombineering of producer strains provide an attractive choice to improve the production titers of structurally complex natural products by microbial fermentation (Zhang et al., 2016).

Exploring the biology of secondary metabolites production in actinomycetes through genetics has provided a foremost share to our current knowledge. Dramatic and sustained increase in understanding the genetics and enzymology of secondary metabolites biosynthesis in actinomycetes, especially Streptomyces throughout the 1990s have also facilitated endurance of natural product search in this admirable bacterial group. As a noteworthy foundation, S. coelicolor A3(2) has genetically been recognized as a model for the actinomycetes, and the whole genome was announced with versatile in vivo and in vitro genetics (Bentley et al., 2002). The genome analysis of $S$. coelicolor A3(2) has revealed the abundance of previously uncharacterized gene clusters, metabolic enzymes, particularly those likely to be involved in the production of natural products. As a latest accomplishment, the marine actinomycete genus Salinispora has been established as a robust model organism for natural product research (Jensen et al., 2015). It has remarkable biosynthetic capacities with 17 diverse biosynthetic pathways of which only four had been linked to their respective products.

The genome information of cultured and uncultured actinomycetes is being promptly updated. Over 1304 actinomycetes genome have been reported as on March 2016 and with the advent of molecular genetics and next-generation genome analysis rapid submissions are expected in near future. Analyses of genomes of actinomycetes have revealed that numerous 'cryptic' or 'orphan' biosynthetic gene clusters with the potential to direct the production of an ample number of novel, structurally diverse natural products (Challis, 2014; GomezEscribano et al., 2016). Subsequently, mining of actinomycetes genome has sketched new directions into the ongoing drug discovery efforts. One such approach has been to mine a collection of 10,000 actinomycetes for novel phosphonic acids, and have laid an intriguing foundation for rapid, large-scale discovery of other classes of natural products (Ju et al., 2015).

Improvements made in bioinformatics methods, particularly specific for natural product gene cluster identification and functional prediction aids in the processing of bulk genomic data of actinomycetes (Alam et al., 2011; Doroghazi et al., 2014; Abdelmohsen et al., 2015). However, sufficient insights into the biology and ecology of antibiotic production are needed to understand the precise triggers and cues required to activate silent gene clusters (Abdelmohsen et al., 2015; Kolter and van Wezel, 2016).

As a great breakthrough, the advent of RNA-guided DNA editing technology Clustered Regularly Interspaced Short Palindromic Repeats (CRISPRs)/Cas9 substantially promises for application to genome modification in biosynthetic gene clusters of actinomycetes (Huang et al., 2015). Obviously, this molecular tool can be used in the engineering of non-model native hosts to heterologous production hosts for the biosynthesis of desired natural products. Continued technological and conceptual advances in engineering microbial hosts will open up opportunities to fully explore and harness Nature's immensely diverse chemical repertoire (Zhang et al., 2016).

\section{FUTURE PERSPECTIVES}

Actinomycetes have been recognized as a premier source of biopharmaceuticals especially antibiotics over several decades. Our universe is rich of diverse unexplored and underexplored environments that could be considered for isolation of novel members of actinomycetes. This could amend our actinomycetes repository with a continuous supply of novel biosynthetic gene clusters and natural product scaffolds on which current research reorient on. Continued advances in genomics and metabolomics reserve a nextgeneration natural products research and unwrap the wider opportunities on the exploitation of actinomycetes that represent an important asset for the discovery of pharmaceutically valuable compounds. The technological and conceptual advances will drive a transition of "searching for desired natural products" to "designing for desired products" from actinomycetes. Through this article, it is evinced that despite an interim decline in actinomycetes research, new avenues are open now and seek the active attention of researchers throughout the world. Those countries well endowed with the natural resources may deem to fund microbial natural products research especially actinomycetes research for extending the inventions of novel antibiotics of industrial 
significance to triumph the escalating microbial resistance and infectious diseases.

\section{AUTHOR CONTRIBUTIONS}

All authors listed, have made substantial, direct and intellectual contribution to the work, and approved it for publication.

\section{FUNDING}

Council of Scientific and Industrial Research (CSIR; http://www.csir.res.in/), Government of India; Project reference:

\section{REFERENCES}

Abdelmohsen, U. R., Grkovic, T., Balasubramanian, S., Kamel, M. S., Quinn, R. H., and Hentschel, U. (2015). Elicitation of secondary metabolism in actinomycetes. Biotechnol. Adv. 33, 798-811. doi: 10.1016/j.biotechadv. 2015.06.003

Alam, M. A., Medema, M. M., Takano, E., and Breitling, R. (2011). Comparative genome-scale metabolic modeling of actinomycetes: the topology of essential core metabolism. FEBS Lett. 585, 2389-2394. doi: 10.1016/j.febslet.2011. 06.014

Becerril-Espinosa, A., Freel, K. C., Jensen, P. R., and Soria-Mercado, I. E. (2013). Marine Actinobacteria from the Gulf of California: diversity, abundance and secondary metabolite biosynthetic potential. Antonie Van Leeuwenhoek 103, 809-819. doi: 10.1007/s10482-012-9863-3

Bentley, S. D., Chater, K. F., Cerdeño-Tárraga, A. M., Challis, G. L., Thomson, N. R., James, K. D., et al. (2002). Complete genome sequence of the model actinomycete Streptomyces coelicolor A3 (2). Nature 417, 141-147. doi: $10.1038 / 417141 \mathrm{a}$

Bérdy, J. (2012). Thoughts and facts about antibiotics: where we are now and where we are heading. J. Antibiot. 65, 385-395. doi: 10.1038/ja.2012.27

Challis, G. L. (2014). Exploitation of the Streptomyces coelicolor A3(2) genome sequence for discovery of new natural products and biosynthetic pathways. J. Ind. Microbiol. Biotechnol. 41, 219-232. doi: 10.1007/s10295-013-1383-2

Cheng, C., MacIntyre, L., Abdelmohsen, U. R., Horn, H., Polymenakou, P. N., Edrada-Ebel, R., et al. (2015). Biodiversity, anti-trypanosomal activity screening, and metabolomic profiling of actinomycetes isolated from Mediterranean Sponges. PLoS ONE 10:e0138528. doi: 10.1371/journal.pone. 0138528

Demain, A. L., and Sanchez, S. (2009). Microbial drug discovery: 80 years of progress. J. Antibiot. (Tokyo) 62, 5-16. doi: 10.1038/ja.2008.16

Doroghazi, J. R., Albright, J. C., Goering, A. W., Ju, K.-S., Haines, R. R., Tchalukov, K. A., et al. (2014). A roadmap for natural product discovery based on large-scale genomics and metabolomics. Nat. Chem. Biol. 10, 963-968. doi: 10.1038/nchembio.1659

Forner, D., Berrué, F., Correa, H., Duncan, K., and Kerr, R. G. (2013). Chemical dereplication of marine actinomycetes by liquid chromatographyhigh resolution mass spectrometry profiling and statistical analysis. Anal. Chim. Acta 805, 70-79. doi: 10.1016/j.aca.2013.10.029

Gomez-Escribano, J. P., Alt, S., and Bibb, M. J. (2016). Next Generation sequencing of Actinobacteria for the discovery of novel natural products. Mar. Drugs 14:E78. doi: 10.3390/md14040078

Guo, X., Liu, N., Li, X., Ding, Y., Shang, F., Gao, Y., et al. (2015). Red soils harbor diverse culturable actinomycetes that are promising sources of novel secondary metabolites. Appl. Environ. Microbiol. 81, 3086-3103. doi: 10.1128/AEM. 03859-14

Hou, Y., Braun, D. R., Michel, C. R., Klassen, J. L., Adnani, N., Wyche, T. P., et al. (2012). Microbial strain prioritization using metabolomics tools for the discovery of natural products. Anal. Chem. 84, 4277-4283. doi: $10.1021 / \mathrm{ac} 202623 \mathrm{~g}$
BSC0106-BioprosPR, and HRDG/CSIR-Nehru PDF LS/EMR$\mathrm{I} / 01 / 2015$.

\section{ACKNOWLEDGMENTS}

CSIR-CSMCRI Communication No.: PRIS-068/2016. The Council of Scientific and Industrial Research (CSIR; www.csir.res.in), Government of India (BSC0106-BioprosPR) is gratefully acknowledged. The CSIR is further acknowledged by PJ for the funding through CSIR-Nehru Postdoctoral Research Fellowship (HRDG/CSIR-Nehru PDF LS/EMR$\mathrm{I} / 01 / 2015)$, to his research on actinomycetes and their secondary metabolism.

Huang, H., Zheng, G., Jiang, W., Hu, H., and Lu, Y. (2015). One-step high-efficiency CRISPR/Cas9-mediated genome editing in Streptomyces. Acta Biochim. Biophys. Sin. (Shanghai) 47, 231-243. doi: 10.1093/abbs/ gmv007

Jensen, P. R., Moore, B. S., and Fenical, W. (2015). The marine actinomycete genus Salinispora: a model organism for secondary metabolite discovery. Nat. Prod. Rep. 32, 738-751. doi: 10.1039/c4np00167b

Jose, P. A., and Jebakumar, S. R. D. (2013). Phylogenetic appraisal of antagonistic, slow growing actinomycetes isolated from hypersaline inland solar salterns at Sambhar salt Lake, India. Front. Microbiol. 4:190. doi: 10.3389/fmicb. 2013.00190

Ju, K. S., Gao, J., Doroghazi, J. R., Wang, K. K., Thibodeaux, C. J., Li, S., et al. (2015). Discovery of phosphonic acid natural products by mining the genomes of 10,000 actinomycetes. PNAS 112, 12175-12180. doi: 10.1073/pnas.15008 73112

Kolter, R., and van Wezel, G. P. (2016). Goodbye to brute force in antibiotic discovery? Nat. Microbiol. 1:15020. doi: 10.1038/nmicrobiol. 2015.20

Kurtböke, D. I., French, J. R., Hayes, R. A., and Quinn, R. J. (2015). Ecotaxonomic insights into actinomycete symbionts of termites for discovery of novel bioactive compounds. Adv. Biochem. Eng. Biotechnol. 147, 111-135. doi: 10.1007/10_2014_270

Mahajan, G. B., and Balachandran, L. (2012). Antibacterial agents from actinomycetes - a review. Front. Biosci. (Elite Ed) 1, 240-253. doi: 10.2741/ e373

Masand, M., Jose, P. A., Menghani, E., and Jebakumar, S. R. D. (2015). Continuing hunt for endophytic actinomycetes as a source of novel biologically active metabolites. World J. Microbiol. Biotechnol. 31, 1863-1875. doi: 10.1007/s11274-015-1950-y

Matsui, T., Tanaka, J., Namihira, T., and Shinzato, N. (2012). Antibiotics production by an actinomycete isolated from the termite gut. J. Basic Microbiol. 52, 731-735. doi: 10.1002/jobm.201100500

Mohammadipanah, F., and Wink, J. (2016). Actinobacteria from arid and desert habitats: diversity and biological Activity. Front. Microbiol. 6:1541. doi: 10.3389/fmicb.2015.01541

Sun, W., Zhang, F., He, L., Karthik, L., and Li, Z. (2015). Actinomycetes from the south China Sea sponges: isolation, diversity, and potential for aromatic polyketides discovery. Front. Microbiol. 6:1048. doi: 10.3389/fmicb.2015. 01048

Tawfike, A. F., Viegelmann, C., and Edrada-Ebel, R. (2013). Metabolomics and dereplication strategies in natural products. Methods Mol. Biol. 1055, 227-244. doi: 10.1007/978-1-62703-577-4_17

Thornburg, C. C., Zabriskie, T. M., and McPhail, K. L. (2010). Deep-sea hydrothermal vents: potential hot spots for natural products discovery? J. Nat. Prod. 73, 489-499. doi: 10.1021/np900662k

Wu, C., Zhu, H., van Wezel, G. P., and Hae Choi, Y. (2016). Metabolomicsguided analysis of isocoumarin production by Streptomyces species MBT76 and biotransformation of flavonoids and phenylpropanoids. Metabolomics 12:90. doi: 10.1007/s11306-016-1025-6 
Zhang, M. M., Wang, Y., Anga, E. L., and Zhao, H. (2016). Engineering microbial hosts for production of bacterial natural products. Nat. Prod. Rep. 33, 963-987. doi: 10.1039/C6NP00017G

Conflict of Interest Statement: The authors declare that the research was conducted in the absence of any commercial or financial relationships that could be construed as a potential conflict of interest.
Copyright (c) 2016 Jose and Jha. This is an open-access article distributed under the terms of the Creative Commons Attribution License (CC BY). The use, distribution or reproduction in other forums is permitted, provided the original author(s) or licensor are credited and that the original publication in this journal is cited, in accordance with accepted academic practice. No use, distribution or reproduction is permitted which does not comply with these terms. 